OPEN ACCESS

Edited by:

Natalia Salvadores, Universidad Mayor, Chile

Reviewed by:

Oliver Schmitt,

University of Rostock, Germany

Ao Zhang,

Emory University, United States

*Correspondence:

Xiaoli Liu

xiaoli@bnu.edu.cn

Received: 14 April 2021

Accepted: 17 May 2021

Published: 14 June 2021

Citation:

Shi K, Liu X, Hou L, Qiao D and

Peng Y (2021) Exercise Improves

Movement by Regulating the Plasticity

of Cortical Function in

Hemiparkinsonian Rats.

Front. Aging Neurosci. 13:695108.

doi: 10.3389/fnagi.2021.695108

\section{Exercise Improves Movement by Regulating the Plasticity of Cortical Function in Hemiparkinsonian Rats}

\author{
Kaixuan Shi ${ }^{1,2}$, Xiaoli Liü ${ }^{2 *}$, Lijuan $\mathrm{Hou}^{2}$, Decai Qiao ${ }^{2}$ and Yuan Peng ${ }^{1}$ \\ ${ }^{1}$ Department of Physical Education, China University of Geosciences, Beijing, China, ${ }^{2}$ College of Physical Education and \\ Sports, Beijing Normal University, Beijing, China
}

Aberrant cortical spike-local field potential (LFP) coupling leads to abnormal basal ganglia activity, disruption of cortical function, and impaired movement in Parkinson's disease (PD). Here, the primary motor cortex mediated plasticity mechanism underlying behavioral improvement by exercise intervention was investigated. Exercise alleviates motor dysfunction and induces neuroplasticity in PD. In this study, Sprague-Dawley (SD) rats were injected with 6-hydroxydopamine (6-OHDA) to induce unilateral nigrostriatal dopamine depletion. Two weeks later, a 4-week exercise intervention was initiated in the PD + exercise (Ex) group. Multichannel recording technology recorded spikes and LFPs in rat motor cortices, and balanced ability tests evaluated behavioral performance. The balanced ability test showed that the total crossing time/front leg error/input latency time was significantly lower in $\mathrm{PD}+$ Ex rats than in $\mathrm{PD}$ rats $(P<0.05)$. Scalograms and LFP power spectra indicated increased beta-range LFP power in lesioned hemispheres, with exercise reducing LFP power spectral density. Spike-triggered LFP waveform averages showed strong phase-locking in PD motor cortex cells, and exercise reduced spike-LFP synchronization. Our results suggest that exercise can suppress overexcitability of LFPs and minimize spike-LFP synchronization in the motor cortex, leading to motor-improving effects in PD.

Keywords: Parkinson's disease, exercise, motor cortex, local field potential, synchronization

\section{INTRODUCTION}

Parkinson's disease (PD) is a progressive neurodegenerative disease characterized by cell death of dopaminergic neurons in the basal ganglia (BG) (Kalia and Lang, 2015). Development of motor impairments, including bradykinesia, rest tremor, rigidity, and lack of coordination, have been associated with exaggerated synchronized oscillation of the beta band $(13-35 \mathrm{~Hz})$ in the corticostriatal circuit of patients with PD (Jankovic, 2008; Tinkhauser et al., 2018). These cortical beta oscillations, which may reflect active inhibition of movement and are related to maintenance of postural tone, are abnormally enhanced with dopamine (DA) depletion and coincide with the emergence of akinesia and bradykinesia (Pogosyan et al., 2009; Leventhal et al., 2012). Furthermore, after levodopa administration or deep brain stimulation (DBS), electrophysiological signal acquisition from the motor cortex and BG of PD patients shows reductions in this coherent beta frequency activity, with motor improvement (Kuhn et al., 2008; Babiloni et al., 2019; Wiest et al., 2020). Unfortunately, these pharmacological and surgical therapies often lead to side effects, including levodopa-induced dyskinesia (Huot et al., 2013; Antosik-Wojcinska et al., 2017). 
Exercise can complement pharmacological therapies in these patients and has been used for rehabilitative management of PD since the 1990s (Duchesne et al., 2015; Pedersen and Saltin, 2015; Fox et al., 2018). While studies of exercise and physical therapy in PD have demonstrated clear improvements in motor performance, substantially less is known about the functional neuroplasticity resulting from long-term exercise interventions (Jakowec et al., 2016; Ferrazzoli et al., 2018). Thus, to explore the potential mechanisms underlying exercise-associated improvements in $\mathrm{PD}$, cortical spikes and local field potentials (LFPs) were recorded simultaneously in control and lesioned rats (created by 6-hydroxydopamine [6-OHDA] injection into the medial forebrain bundle [MFB]), and spike-LFP relationships were investigated.

\section{MATERIALS AND METHODS}

\section{Animals and Experimental Procedures}

Sprague-Dawley (SD) rats (male, 230-250 g, 8 weeks old, from Beijing Vital River Laboratory Animal Technological Company, Beijing, China) were housed in a controlled environment, with a 12/12-h light/dark cycle (lights on at 18:00) and ad libitum access to food and water. The Beijing Normal University Committee for Animal Care approved all experimental procedures, and this study complied with the guidelines set forth by the National Institutes of Health.

All rats were initially randomly assigned to three groups: (1) control group $(n=11)$, (2) PD group $(n=14)$, and (3) PD with exercise (PD + Ex) group $(n=14)$. As shown in Figure 1, rats in the $\mathrm{PD}+\mathrm{Ex}$ group were placed on a treadmill, and all rats practiced using the automatic footfault equipment to familiarize them with the study procedures. The baseline behavioral level was evaluated after 1 week of habituation.

Then, animals in the control and PD groups (including the $\mathrm{PD}$ and $\mathrm{PD}+$ Ex groups) were administered vehicle or 6OHDA, respectively. At 7 days after surgery, rats were tested for their rotational behavior using apomorphine (APO; $0.5 \mathrm{mg} / \mathrm{kg}$, subcutaneous (s.c.); Sigma-Aldrich, St. Louis, MI, USA), with a total net number of rotations of $\geq 100$ per 30 min indicating rats represented a model of Parkinson's disease, as described previously (Shi et al., 2017a). Subsequently, electrophysiological recording and automatic foot-fault testing were performed once per week. Starting during Week 1, rats in the PD + Ex group began a 4-week exercise intervention. At the end of Week 4, histological localization of electrodes and biochemical assays were performed.

\section{Nigrostriatal Lesions and Electrode Implantation}

Each rat was anesthetized with chloral hydrate $(350 \mathrm{mg} / \mathrm{kg})$ and then placed on a stereotaxic frame (Stoelting, Chicago, IL, USA) fitted with atraumatic ear bars to level the skull in the dorsalventral plane (mouth bar was set at $-3.3 \mathrm{~mm}$ ). A small hole was made in the skull at the right MFB coordinate (Paxinos and Watson, 2007) (AP: $-4.3 \mathrm{~mm}, \mathrm{ML}:-1.5 \mathrm{~mm}$, and DV: 7.6$7.8 \mathrm{~mm}$ ), and an $8-\mu \mathrm{g}$ solution of 6-OHDA ( $4 \mu \mathrm{L}$ in total, per $\mu \mathrm{L}$ in $0.2 \%$ ascorbic acid and $0.9 \%$ saline, Sigma-Aldrich) was injected into this hole to lesion nigral dopaminergic cells, with an injection rate of $1 \mu \mathrm{L} / \mathrm{min}$ and slow syringe withdrawal (1 $\mathrm{mm} / \mathrm{min}$ ) over $5 \mathrm{~min}$. Control animals received an equal volume of a solution of $0.2 \%$ ascorbic acid and $0.9 \%$ saline in the same manner.

Electrode implantation was conducted after 6-OHDA or vehicle injection. A chronic 16-channel microwire electrode array, which consisted of $4 \times 4$ rows of electrodes with an additional ground wire serving as a local reference (Stablohm 675, MicroProbes, USA; tip diameter $=35 \mu \mathrm{m}$, spacing $=$ $300 \mu \mathrm{m})$, was implanted into the right primary motor cortex (M1) layers 5/6 (AP: $1.8 \sim 3.0 \mathrm{~mm}$, ML: $2.3 \sim 3.3 \mathrm{~mm}$, DV: $1.4-$ $1.6 \mathrm{~mm}$ ) (Paxinos and Watson, 2007). Finally, five stainless steel screws were anchored to the skull and wrapped with a reference wire. Dental cement (A-M Systems, WA, USA) was added to secure the skull screws.

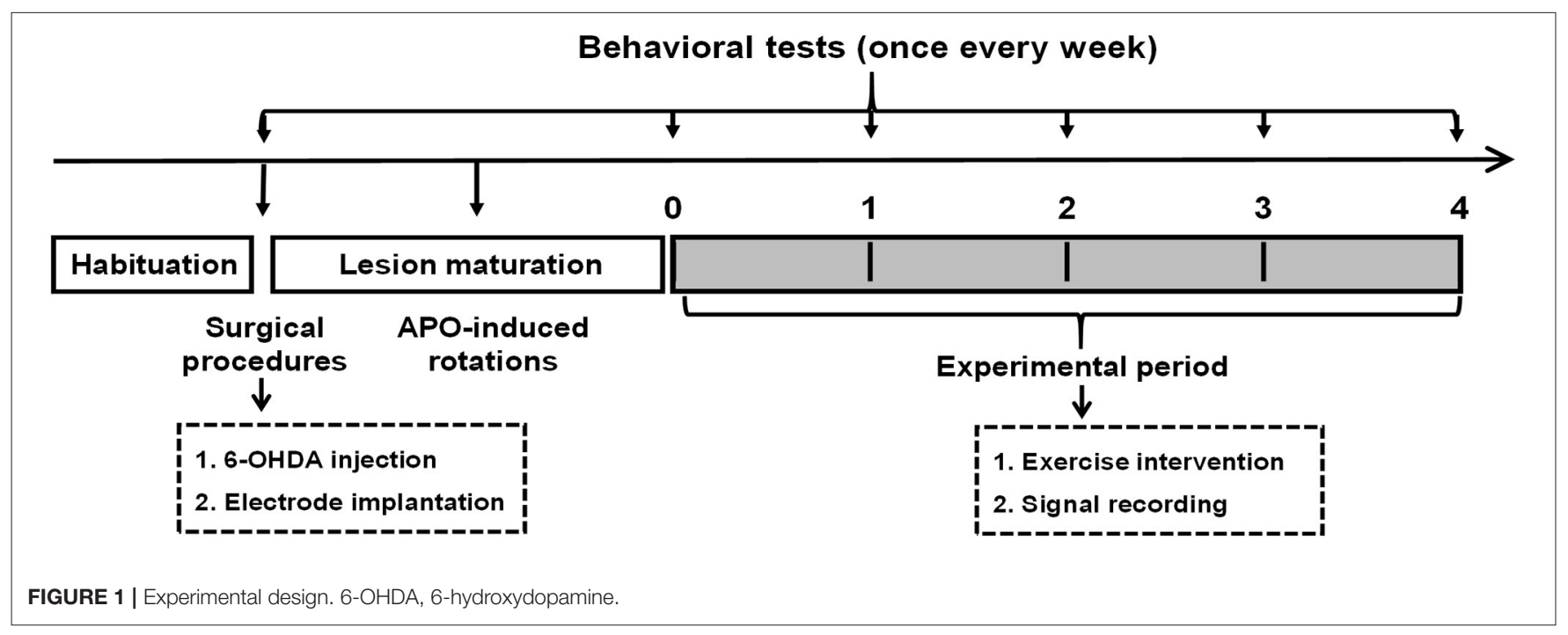




\section{Exercise Protocol}

Before undergoing surgery, all animals were adapted to the treadmill with a speed limit of $11 \mathrm{~m} / \mathrm{min}$. Two weeks after 6OHDA injections, the sustained mandatory treadmill protocol $(11 \mathrm{~m} / \mathrm{min}, 30 \mathrm{~min}$ per day) was performed 5 days/week for 4 weeks (Tajiri et al., 2010; Chen et al., 2017) without electrical shock and at a $0^{\circ}$ inclination. Meanwhile, rats in both the control and PD groups were placed on an unmoving treadmill for $30 \mathrm{~min}$ at a time.

\section{Behavioral Testing}

To evaluate sensorimotor function, an automatic foot fault test was carried out before and after the 6-OHDA lesion was induced. One week before surgery, rats were placed at the departure box and trained to walk spontaneously across a horizontal scale with 77 bars to the arrival box. Rats underwent three training trials per day for 3 days, after which they could reliably walk from the departure box to the arrival box with fewer than five errors. Infrared beams and a sensor device (LocotronicH, Bioseb, Vitrolles, France; $\mathrm{L} \times \mathrm{W} \times \mathrm{H}: 128 \mathrm{~cm} \times 28 \mathrm{~cm} \times 20 \mathrm{~cm})$ detected the movement of rats along the length of the ladder and were used to recognize and record the exact position and duration of errors in motor coordination (missteps or paw slips between any two rungs). The included software identified input latency times, front errors, and total crossing times. Each rat performed the test two times per day on Weeks $0,1,2,3$, and 4 , with a rest of 5 min between two trials.

\section{Electrophysiological Data Acquisition and Analysis}

The raw neural signals from each microwire of the electrode were amplified and recorded using the Cerebus ${ }^{\mathrm{TM}}$ 128-Channel Data Acquisition System (Cyberkinetics Inc., Salt Lake City, UT, USA). Low-pass, filtered, LFP channels were amplified by $1000 \times$, sampled at $1 \mathrm{kHz}$, and filtered under $250 \mathrm{~Hz}$. Meanwhile, high-pass, filtered, waveform channels were amplified by $20,000 \times$, digitized at $30 \mathrm{kHz}$, and bandpass-filtered between 250 and $5000 \mathrm{~Hz}$. Spike detection and sorting were carried out automatically in real-time using the Centre software of the Cerebus $^{\text {TM }}$ system. Simultaneously, the rat's automatic activities were tracked and recorded by the Neuromotive ${ }^{\mathrm{TM}}$ system (Cyberkinetics Inc., Salt Lake City, UT, USA). Videotaped motor behavior was used to identify a sedentary behavioral state.

All recorded data were stored for additional offline analyses. Off-line Sorter software version 4.2.0 (Plexon, Dallas, TX, USA), Neuroexplorer software version 5 (Nex Technologies, Littleton, MA, USA), and custom-written MATLAB routines were used for these analyses.

\section{Power Spectral Density Analysis of LFPs}

Epochs of 300 LFP recordings (according to the rat's behavioral rest state) were captured, and the trapped filter was set to $50 \mathrm{~Hz}$ to remove power line interference and other high-frequency noises. Next, the spectrum value was normalized as the log of the raw power spectral density (PSD) from 0 to $80 \mathrm{~Hz}$, which was constructed using a fast Fourier transform (FFT) with a based analysis and a 1-s moving window. The PSD and frequency blocks were set at 1,024 and 512 points at $0.2 \mathrm{~Hz}$, respectively.

\section{Spike Sorting and Cell-Type Classification}

Spike waveforms recorded from M1 were required to satisfy the following conditions: (1) the threshold was three times the standard deviation; (2) the signal-to-noise ratio was more than 2.5; and (3) the interspike interval (ISI) histogram reflected spikes that did not occur within the assumed refractory period of $1.2 \mathrm{~ms}$. Next, single-unit spikes were sorted using a principal component analysis (PCA) and a K-means cluster analysis to measure action potential widths and the frequency of spike trains. PC1 and PC2 ( $x$ and $y$ axes) were plotted against time (z-axis) to isolate each unit in 2D and 3D PC space, as shown in Figure 2A. The F, J3, and Davis-Bouldin (DB) statistics were also calculated to determine whether the classification was qualified. Figure 2B shows the three identified units ( $a, b$, and $c$ ) according to the spike waveform characteristics (top) and the ISI histograms (bottom).

Based on several studies comparing morphology with electrophysiological characteristics in M1, spikes were classified into two different neuronal subpopulations (Li et al., 2012; Lacey et al., 2014). One neuron type exhibited relatively long action potential widths $(0.5-0.8 \mathrm{~ms})$ and low discharge frequencies $(<10 \mathrm{~Hz})$ and were categorized as pyramidal neurons (PNs). Conversely, another neuron type had shorter action potential widths $(0.2-0.5 \mathrm{~ms})$ and higher spontaneous discharge frequencies $(8-45 \mathrm{~Hz})$ and was presumed to be interneurons (INs).

\section{Spike-Triggered Waveform Analysis}

Spike-triggered LFP waveform averages (STWA) were calculated to assess the degree of entrainment between spontaneous spike potentials of distinct neurons and LFP oscillations (Figure 3). LFPs were bandpass-filtered (beta band, 10-30 Hz) using the MATLAB signal-processing toolbox. STWAs were calculated as follows: $0 \mathrm{~ms}$ denoted the spike timestamp for a certain spike train, and filtered beta bands were averaged from two 200-ms epochs (unshuffled STWA). Subsequently, the original interspike intervals were randomly shuffled to produce different STWA. This process was repeated 1,000 times to provide a mean value for the beta band (shuffled STWA). Spikes were deemed to be related to beta oscillations when the unshuffled STWA exceeded the $96 \%$ confidence interval for the mean of the 1,000 shuffled spike trains at the timestamp $(0 \mathrm{~ms})$. Thus, the percentage of correlated neurons was calculated. The ratio of the mean unshuffled to the mean shuffled STWA was also calculated to estimate the proportion of synchronized neurons. When this ratio was close to 1 , it indicated no phase locking between spikes and beta oscillations (Denker et al., 2011; Brazhnik et al., 2016; Wilson et al., 2018).

\section{Histology and Immunohistochemistry}

After electrophysiological recordings were complete, the rats (control, $n=9$; PD, $n=11 ; \mathrm{PD}+\mathrm{Ex}, n=13$ ) were deeply anesthetized by an intraperitoneal (i.p.) injection of chloral hydrate $(450 \mathrm{mg} / \mathrm{kg})$ and transcardially perfused with 


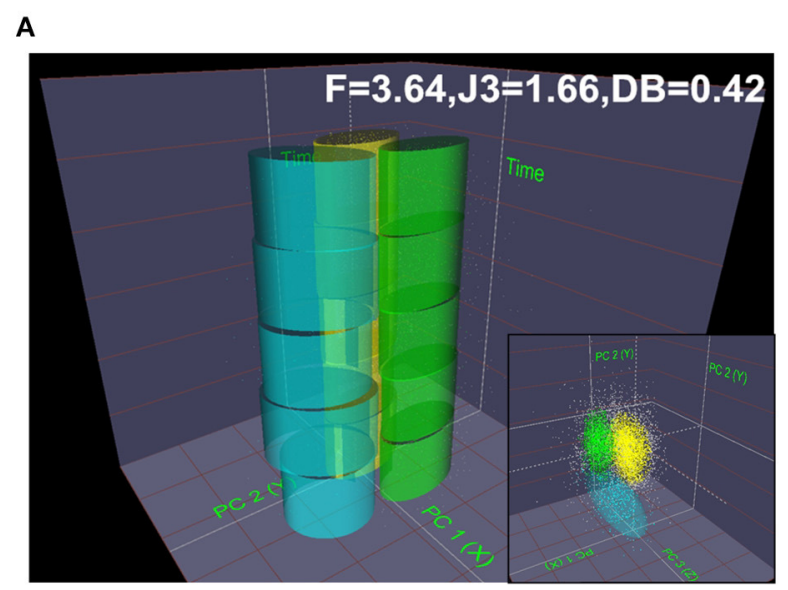

$\mathbf{B}$
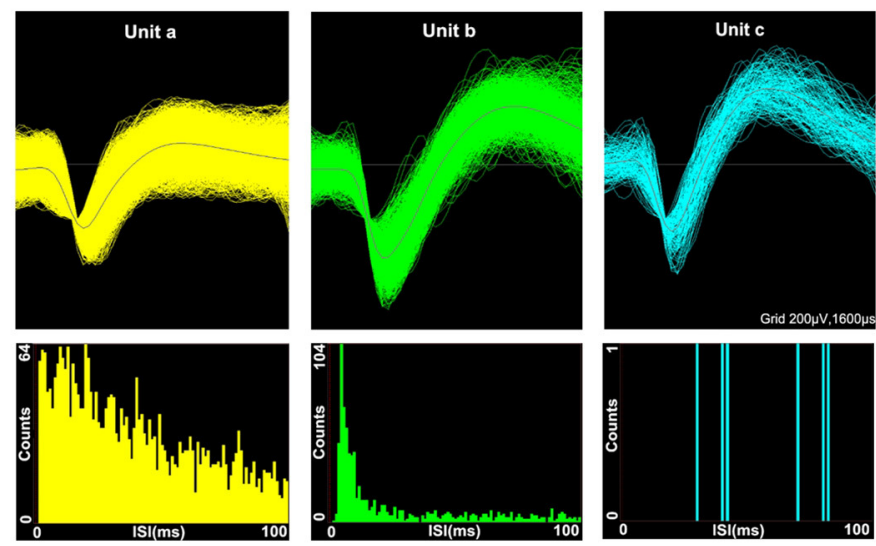

FIGURE 2 | Diagram of offline unit sorting by principal component analysis (PCA) and the quality metrics of F, J3, and Davis-Bouldin (DB). Spikes were sorted using PCA in 2D and 3D PC spaces, (A) and the three units (a, b, c) were well-isolated based on their respective spike waveforms and interspike interval (ISI) histograms (B).

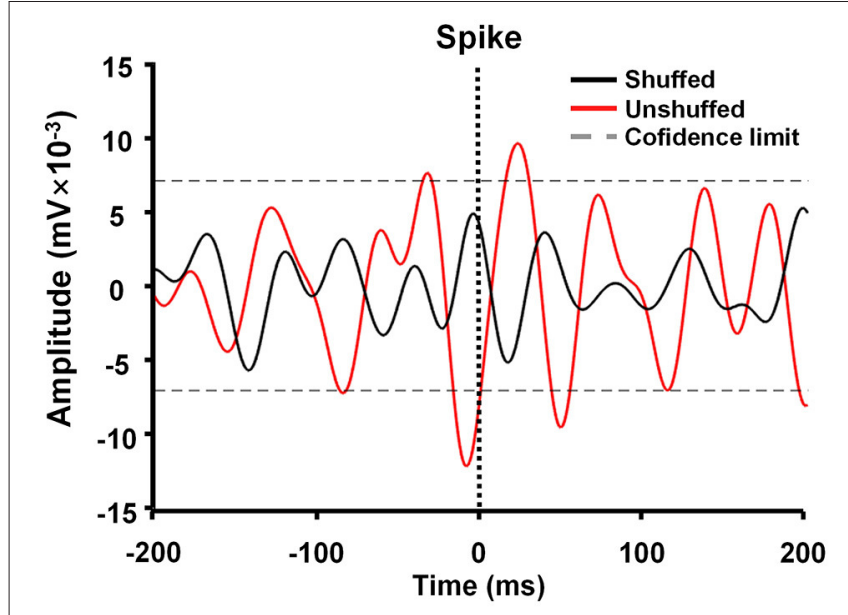

FIGURE 3 | Example of M1 spike-triggered 10-30-Hz waveform averages. The unshuffled spike train (red) exceeded the $96 \%$ confidence interval of the shuffled spike train (black) and represented spike-local field potential (LFP) synchronization in the $10-30 \mathrm{~Hz}$ range.

saline followed by $4 \%$ paraformaldehyde (PFA) in phosphatebuffered saline $(0.1 \mathrm{M}, \mathrm{pH} 7.4)$. Brains were dissected and fixed in $4 \% \mathrm{PFA}$ overnight at $4^{\circ} \mathrm{C}$. Coronal sections were cut at M1 $(50 \mu \mathrm{m})$, the substantia nigra pars compacta (SNc; $30 \mu \mathrm{m})$, and the striatum $(30 \mu \mathrm{m})$ using a freezing microtome. The sections for recording electrode targets were mounted on glass slides and stained with cresyl violet. To assess dopaminergic denervation in PD models, sections from the SNc and striatum were analyzed by tyrosine hydroxylase (TH) immunohistochemistry, as described previously (Shi et al., 2019). The number of TH-immunoreactive neurons in the SNc and the optical density of $\mathrm{TH}$-immunoreactive fibers in the striatum were evaluated. Results were normalized in each rat by values obtained on the right and left sides using the following formula: lesioned/unlesioned.

\section{Data Analysis}

All data are presented as the mean \pm standard error of the mean (SEM). Multiple comparisons were performed by one-way (before vs. after exercise intervention, i.e., "pre- vs. post" comparisons) or two-way (between two different groups) repeated-measures analysis of variance (ANOVA) followed by Student-Newman-Keuls post-hoc testing. All analyses were carried out using SPSS 21.0 (IBM Corp., Armonk, NY, USA) and Sigmaplot 13.0 (Systat Software, Inc, Richmond, CA, USA). A $P$-value of $\leq 0.05$ was considered statistically significant.

\section{RESULTS}

\section{Behavioral Results}

Rats were placed on the automatic foot fault device (Figure 4D), and 6-ODHA-lesioned rats were shown to have deficits in the adjusting step test. In the control and PD groups, the three test indicators showed no significant changes compared to Week $0(P$ $>0.05$, Figures 4A-C). For the total crossing time ( $\%$ baseline), exercise improved the performance of $\mathrm{PD}$ rats by enhancing vigor and reducing the duration of time required to cross the beam at Weeks 2, 3, and 4 (at Weeks 2 and 3, $P<0.05$; at Week 4, $P<0.01$, Figure 4A) compared to Week 0 . Input latency times showed the same trend (at Week 2, $P<0.05$; at Weeks 3 and $4, P<0.01$, Figure 4C), whereas deficits in front leg errors were reduced at Week $3(P<0.05)$ and remained relatively stable at Week $4(P<0.01)$.

\section{Histological and TH-Immunoreactivity Results}

APO-induced rotation showed that 24 rats were successfully modeled in the PD and PD + Ex groups, with net rotations consistent with the criterion for the PD model $(165.43 \pm 17.67$ $\mathrm{r} / 30 \mathrm{~min}>100 \mathrm{r} / 30 \mathrm{~min}$ ). Failed modeling occurred in four rats that had net rotations $<60 \mathrm{r} / 30 \mathrm{~min}$.

Histological staining with cresyl violet confirmed correct placement of the recording electrode array in M1 (layer 5) 


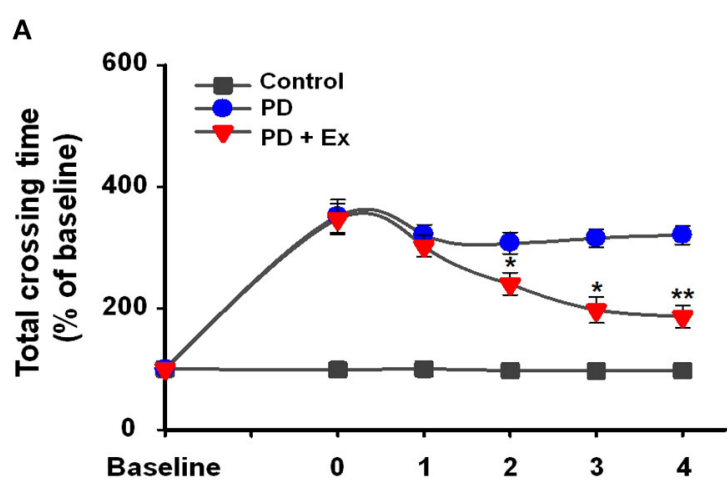

C

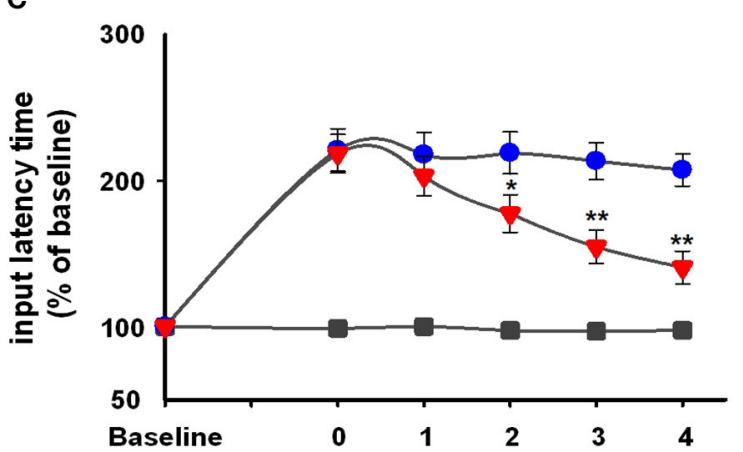

B

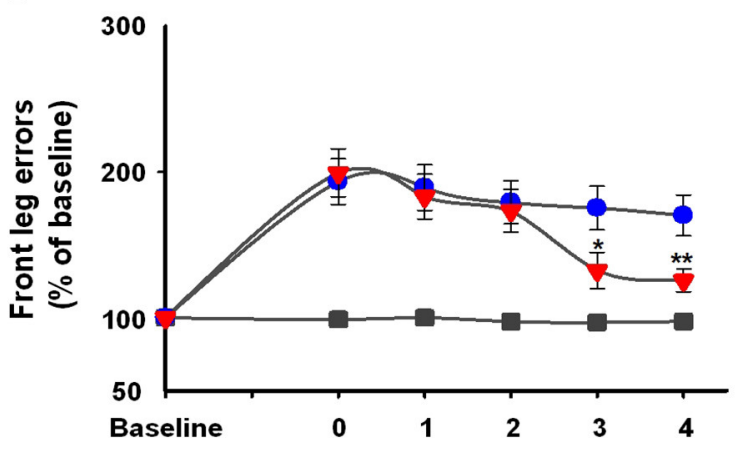

D

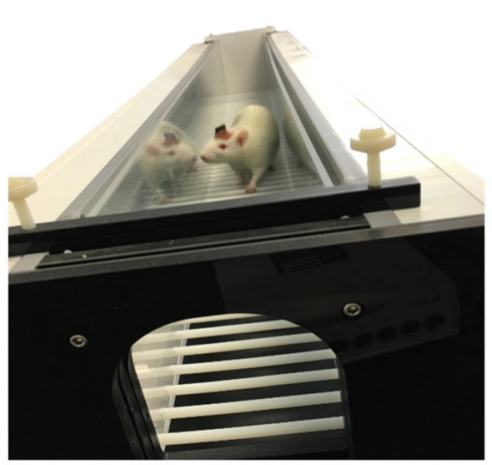

FIGURE 4 | Automatic foot fault test (D) results in the control, Parkinson's disease (PD), and PD + exercise (Ex) groups. Time course of the automatic foot test and the percentages of baseline for total crossing time (A), front leg error (B), and input latency time (C). Compared with the Week 0 standard ${ }^{*}: P<0.05,{ }^{\star *}: P<0.01$.

on coronal sections (Figure 5A). Figures 5B,C shows immunostaining for $\mathrm{TH}$ in rat $\mathrm{SNc}$ and striatum, with obviously lower numbers of $\mathrm{TH}$-immunoreactive cells and fibers on the lesioned side relative to the unlesioned side. Compared with the control group, 6-OHDA-lesioned rats had significantly reduced nigrostriatal projections in both the $\mathrm{PD}$ and $\mathrm{PD}+$ Ex groups $(P$ $<0.01)$. The exercise intervention was unable to significantly reverse the nigrostriatal depletion in these groups $(P>0.05$, Figures 5D,E).

\section{Time-Dependent Changes in Spontaneous Firing Rates in M1 Correlates With the Efficacy of Exercise}

As described in section Spike Sorting and Cell-Type Classification, PNs and INs have distinct electrophysiological characteristics. Based on the electrophysiological properties of spontaneous firing rate and spike width (Figure 6), PNs and INs can be distinguished explicitly. The proportions of these two types of neurons in the study groups are summarized in Table $\mathbf{1 .}$

An intergroup analysis of the spontaneous firing activity of PNs and INs at Week 4 indicated that the mean firing rate was different after induction of 6-ODHA lesions. As shown in Figure 7A, the mean firing rate of PNs was significantly lower in the PD group than in the control group (3.427 \pm 0.703 vs. $2.569 \pm 0.79 \mathrm{~Hz}, P<0.01)$. Compared with the PD group, the mean frequency of PN firing in the PD + Ex group was obviously increased $(3.033 \pm 0.77, P<0.01)$ but was still significantly lower than in the Control group $(P<0.01)$. The average frequency of firing for INs, however, did not significantly differ between the three groups $(P>0.05)$.

Intragroup comparisons of mean firing rates showed that these two types of neurons did not have significant alterations at Weeks $0,1,2,3$, or 4 , either in the control group or PD group $(P>0.05$, Figures 7B,C). For the $\mathrm{PD}+$ Ex group, however, the mean firing rate of PNs was significantly increased from Week 3 to 4 compared with Week $0(P<0.05)$, though there were no changes in INs during exercise training $(P>0.05$; Figure 7D).

\section{Exercise Attenuates Cortical Beta Oscillatory LFP Power in 6-ODHA-Lesioned Rats}

In this study, hemiparkinsonian rats (PD and $\mathrm{PD}+$ Ex groups) exhibited higher cortical LFP power in the $10-30 \mathrm{~Hz}$ range than control rats in the resting state $(P<0.01$ and $P<$ 0.05 , respectively, Figures $\mathbf{8 A}, \mathbf{B}$ ). The PSDs of beta LFPs were significantly higher in the $\mathrm{PD}+$ Ex group than in the $\mathrm{PD}$ group $(P$ $<0.05$, Figure $8 C$ ), and this change was time-dependent, which has been confirmed in our previous study (Shi et al., 2017b).

\section{Exercise Disrupts the Pathological Synchrony Between M1 Neurons and Beta Oscillations}

The spike-LFP relationship was examined in M1 to determine whether changes in spike timing correlated with the increases 
A

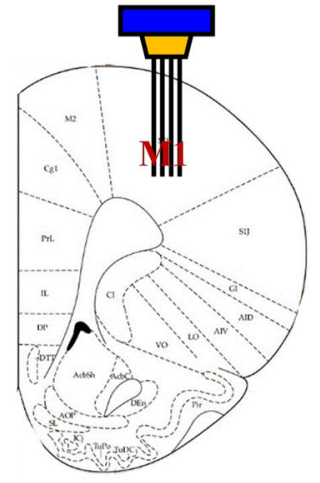

Bregma $2.70 \mathrm{~mm}$

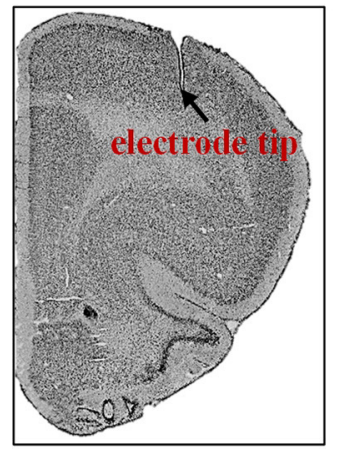

B

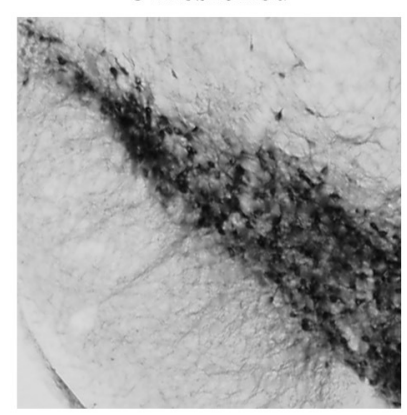

Lesioned

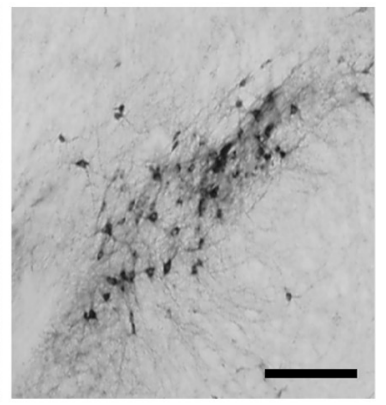

Bar $=\mathbf{5 0} \mu \mathrm{m}$
C

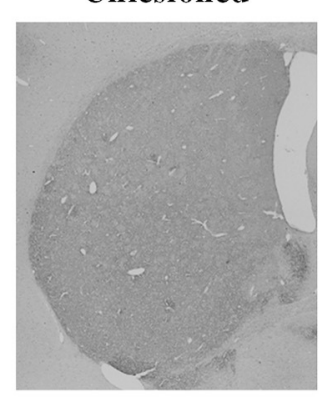

Lesioned

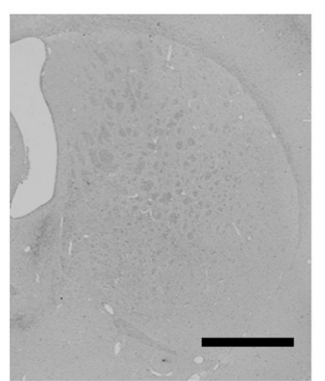

D

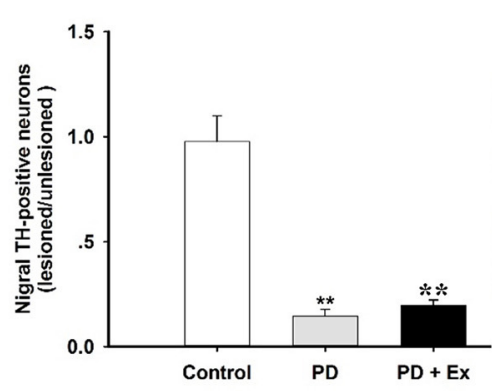

E

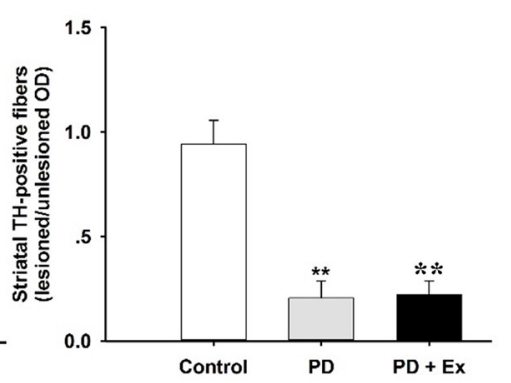

FIGURE 5 | Histological results in the M1 and tyrosine hydroxylase (TH)-immunostaining results in the substantia nigra pars compacta (SNc). The recording electrode site at M1 was confirmed on the coronal section and adapted from Paxinos and Watson (2007) (A). TH-immunostaining results in the SNc (B) and striatum (C) from both unlesioned and lesioned sides. There were significantly lower numbers of TH-positive neurons in the Parkinson's disease (PD) and PD + exercise (Ex) groups than in the Control group (**: $P<0.01)$; however, there was no significant difference between the PD and PD + Ex groups $(P>0.01)(\mathbf{D}, \mathbf{E})$.
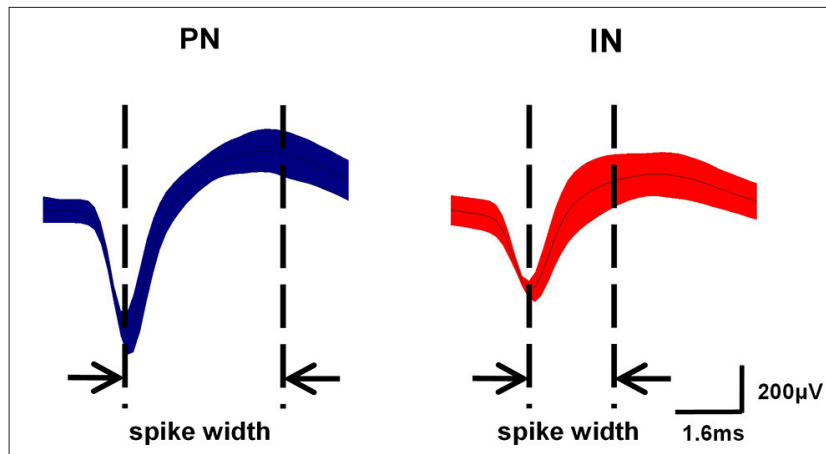

FIGURE 6 | Classification of neurons in M1. Waveform examples of pyramidal neuron (PN) (blue) and interneuron (IN) (red) subtypes in M1. PNs exhibited relatively long action potential widths $(0.5-0.8 \mathrm{~ms})$ and low spontaneous firing rates $(<10 \mathrm{~Hz})$, while INs had shorter action potential widths $(0.2-0.5 \mathrm{~ms})$ and higher spontaneous firing rates $(8-45 \mathrm{~Hz})$.

in LFP beta-range $(10-30 \mathrm{~Hz})$ activity observed in 6-OHDAlesioned rats. As described in section Data Analysis, increases in the LFP power in the $10-30-\mathrm{Hz}$ range in the lesioned hemisphere have been associated with an increased synchronization of spikes and LFP oscillations in the same frequency range.
TABLE 1 | Proportions of pyramidal neurons (PN) and interneurons (IN) in the motor cortices of rats in the control, Parkinson's disease (PD), and Parkinson's disease + exercise $(P D+$ Ex) groups.

\begin{tabular}{lccc}
\hline Group & Total neurons & PN (\%) & IN (\%) \\
\hline Control $(n=9)$ & 113 & $86(76.1 \%)$ & $27(23.9 \%)$ \\
$\operatorname{PD}(n=11)$ & 147 & $104(70.7 \%)$ & $43(29.3 \%)$ \\
PD + Ex $(n=13)$ & 156 & $123(78.8 \%)$ & $33(21.2 \%)$ \\
\hline
\end{tabular}

Figure 9A shows STWA without obvious phase locking, and Figure 9B depicts typical STWA synchronization. The mean ratios of unshuffled-to-shuffled STWA peak-to-trough amplitudes (STWA ratios) of cortical neurons in the $10-30-\mathrm{Hz}$ band were compared between the three groups. Figure 9C shows that the STWA ratio was higher in PD models than in control rats (compared to the control group, $\mathrm{PD}: P<0.01, \mathrm{PD}+\mathrm{Ex}$ : $P<0.05)$. Following the exercise intervention, phase-locking of spikes with beta-band oscillations was reduced in the PD $+\mathrm{Ex}$ group compared to the PD group $(P<0.05)$. In the control group, PNs and INs did not show notable synchronization of spiking with the beta band. The percentage of neurons (both PNs and INs) entrained to STWA was significantly increased 

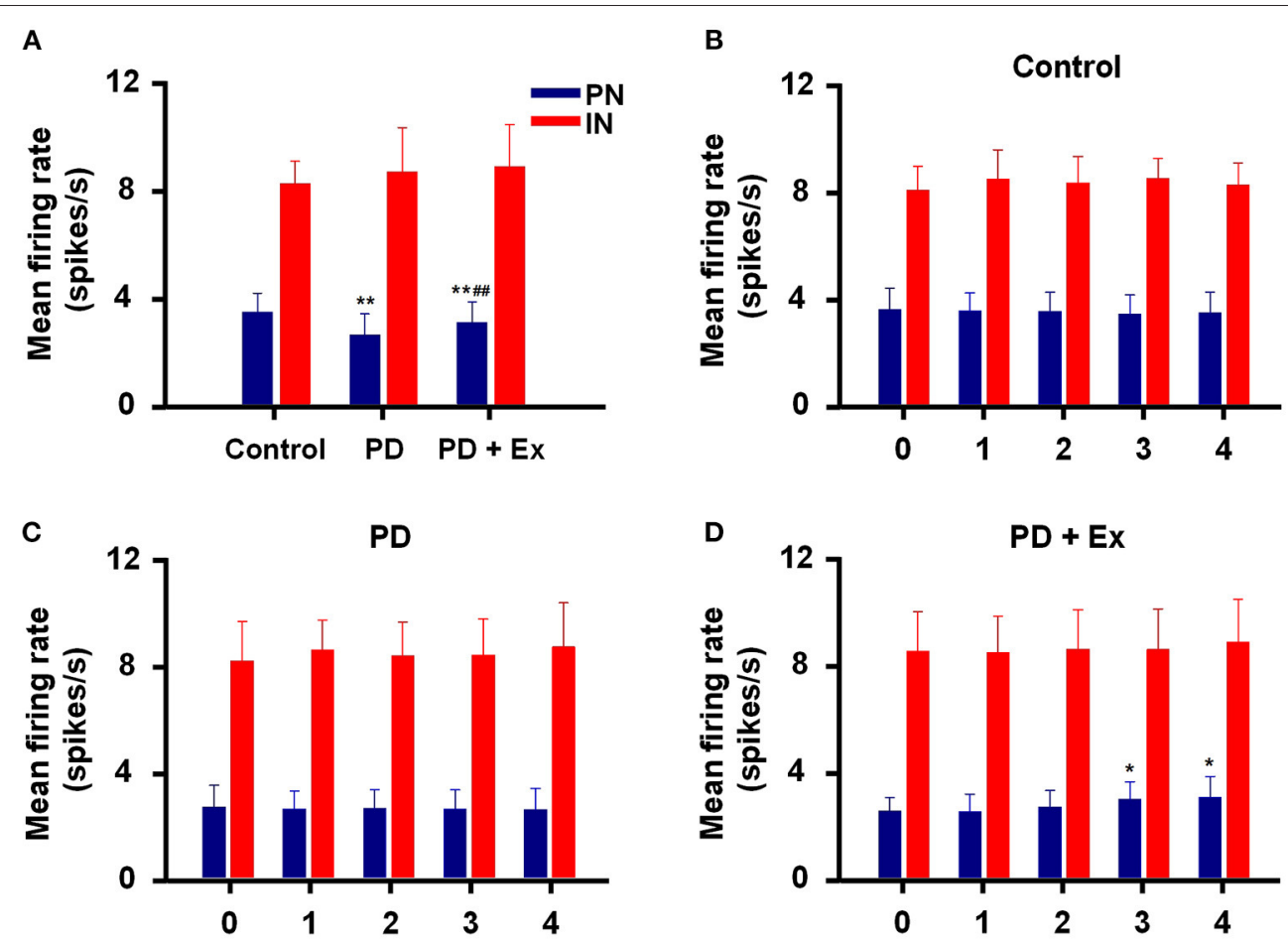

FIGURE 7 | Inter- and intragroup comparisons of the mean firing rates of pyramidal neurons (PNs) and interneurons (INs) in M1. The intergroup analysis of PNs (blue) and INs (red) at Week 4 is shown in (A). The mean firing rates of PNs and INs varied with time in the Control (B), Parkinson's disease (PD) (C), and PD + exercise (Ex) (D) groups. Compared with the Control group or Week 0 data, ${ }^{\star}: P<0.05$, ${ }^{* *}$ : $P<0.01$; and compared with the PD group, \#: $P<0.05$, \#\#: $P<0.01$.
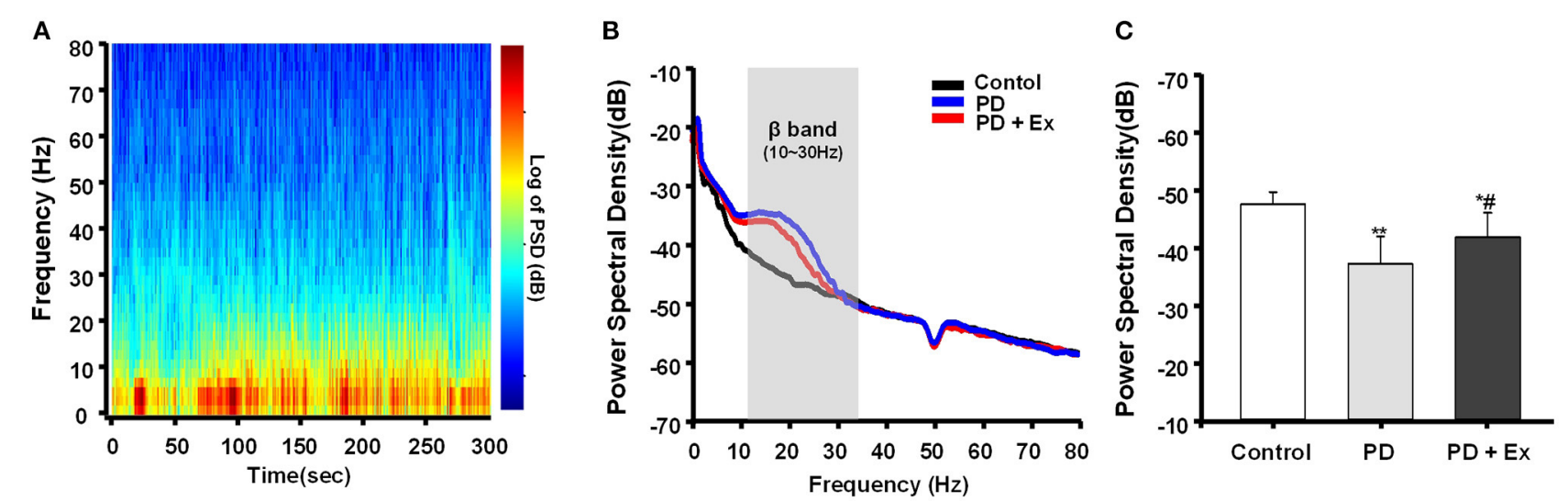

FIGURE 8 | Power spectral density (PSD) of $\beta$ local field potentials (LFPs) recorded in M1. A representative fast Fourier transform (FFT)-based spectrogram depicts the time-frequency spectral power of motor cortex LFPs during 5-min epochs of rest (A). Linear graphs show averaged LFP power (0-80 Hz) spectra for the Control, Parkinson's disease (PD), and PD + exercise (Ex) groups (B), with the gray area showing that the beta band in the 10-30 Hz range of LFP power was significantly increased in 6-hydroxydopamine (6-OHDA)-lesioned rats. The average PSDs of the three groups are shown in (C). The PSD of the beta band was increased in the PD group compared to the Control group. For the PD + Ex group, the PSD of the beta band was decreased compared to the PD group but was still higher than in the Control group. Compared with the Control group, ${ }^{*}: P<0.05,{ }^{* *}: P<0.01$; and compared with the PD group, \#: $P<0.05$.

in PD model rats compared to control rats (compared to the control group, percent PNs and INs in PD: $P<0.01$, percent PNs and INs in PD + Ex: $P<0.01)$. After the exercise intervention, the percentage of these neurons was smaller than in the PD group (percent PNs: $P<0.01$; percent INs: $P<0.05$;
Figure 9D). The polar plots in Figure 9E show the distribution of STWA phases from spike trains in the M1 with cortical LFP $10-30-\mathrm{Hz}$ oscillations. The phase angles between spikes and beta oscillations appeared random in control rats; however, the STWA of spike trains in PD rats showed strong phase-locking at 


\section{A}

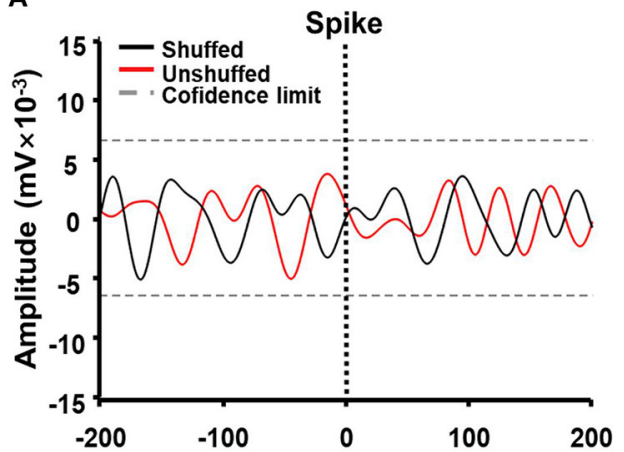

C

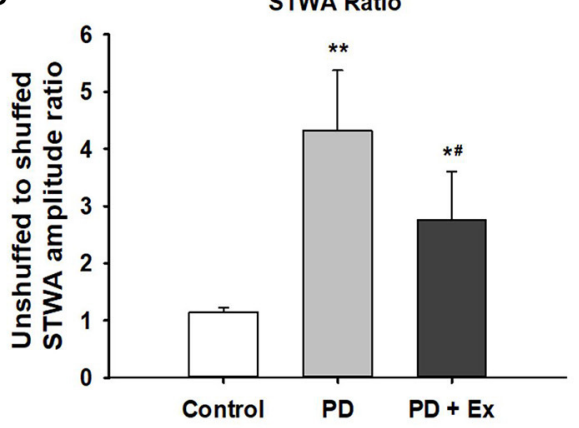

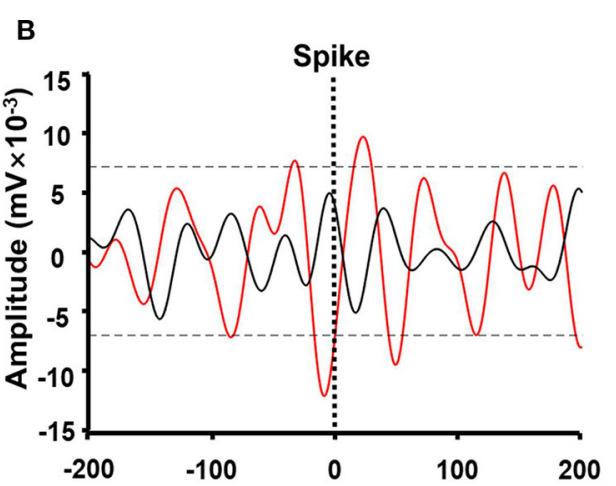

D Pencent neurons enntrained to STWA

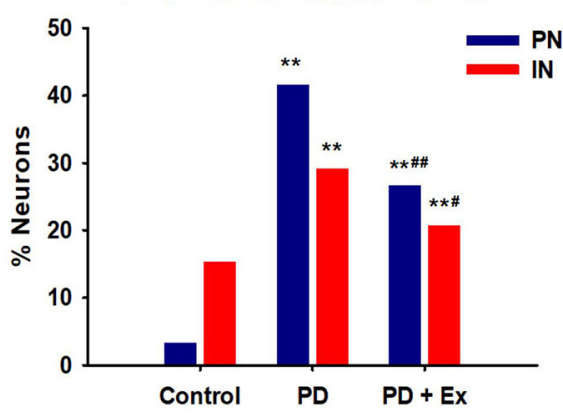

E

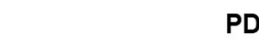

PD

$P D+E x$

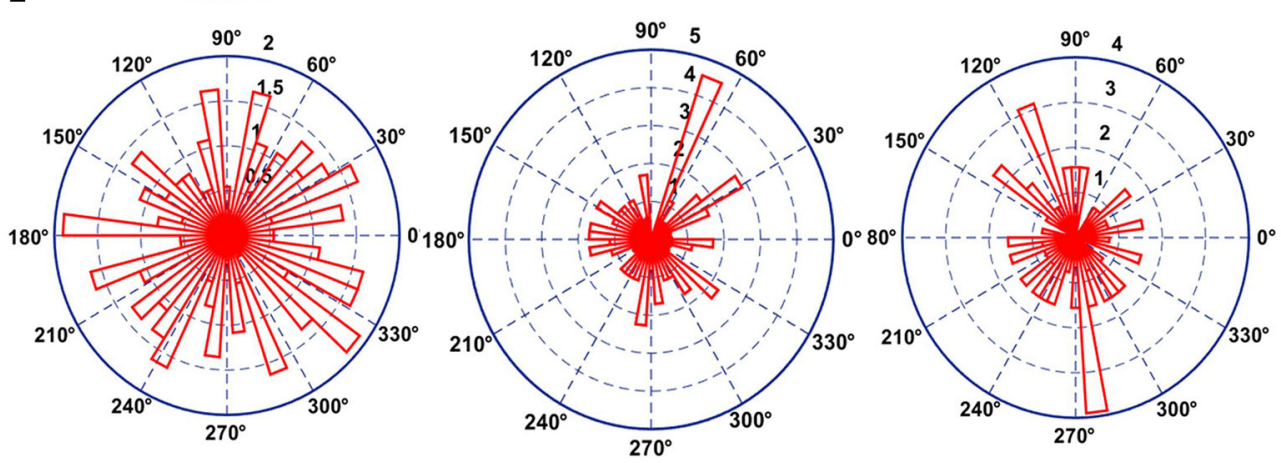

FIGURE 9 | Phase locking of cortical spiking with 10-30 Hz local field potentials (LFPs) in the three groups. Unsynchronized spike-triggered LFP waveform averages (STWA) (A). Example STWA for a typical cortical spike train paired with an LFP in the 10-30 Hz range (B). The ratio of STWA-based unshuffled-to-shuffled spike trains indicating cortical spike-LFP synchronization (C). Bar graphs show the proportions of pyramidal neurons (PNs) and interneurons (INs) significantly synchronized with 10-30 Hz LFP oscillations (D). To assess STWA, mean phase angles between spikes and cortical LFP oscillations were significantly clustered around different phases of cortical 10-30 Hz LFP oscillations, as the polar plots show in (E). Compared with the Control group, ${ }^{*}: P<0.05,{ }^{* *}$ : $P<0.01$; and compared with the PD group, $\#: P<0.05 ; \# \#: P<0.01$

about $60^{\circ}$. The STWA of cortical neurons in the PD + Ex group seemed to be relatively scattered but still presented with obvious phase-locking consistent with the STWA ratio results.

\section{DISCUSSION}

Previous studies have demonstrated that exercise training can modulate activity in the cortical motor areas of PD patients, as well as in animal models of parkinsonism
(Wang et al., 2015; Alberts et al., 2016; Ginanneschi et al., 2021). In this study, multichannel electrode arrays were implanted into the M1, and spike trains of neurons and LFPs were recorded and analyzed in hemiparkinsonian rats during a therapeutically effective exercise intervention. This approach allowed us to address several key questions related to the role of functional changes in $\mathrm{M} 1$ on $\mathrm{PD}$, providing insights into the mechanism of exercise-induced neuroplasticity. 
This study identified increases in spiking discharges and beta-band oscillations, which was consistent with the results of previous studies conducted in humans and PD animal models, including rodents and non-human primates (Hammond et al., 2007; Zhuang et al., 2018; Holt et al., 2019). Abnormal spike and beta LFP activity at various levels of the cortical-basal gangliathalamocortical circuit have been observed in PD individuals (patients and animal models), though the origin of these changes remains unknown (Goldberg et al., 2004; Dejean et al., 2008; Stein and Bar-Gad, 2013). It is generally hypothesized that pathological changes in the motor cortex may contribute to the motor dysfunction observed in parkinsonian conditions. Li et al. (2012) have confirmed that exaggerated spike trains and beta oscillations can deteriorate motor control and lead to the development of akinesia and bradykinesia. Furthermore, several studies focusing on the mechanisms of subthalamic nuclei DBS or L-3, 4-dihydroxyphenylalanine (L-DOPA) treatment have shown that L-DOPA administration reduces the cortical power in the beta range and that STN-DBS induces reductions in the synchronization of oscillations in the cortex-basal ganglia circuit.

Although M1 is a crucial basal ganglia output area that transforms neurophysiological signals into motion instructions, there have only been a few previous studies related to exercise-induced plasticity of cortical neural activity (e.g., electrophysiological evaluations) at the cellular level in PD patients (Bonavita, 2020; Feng et al., 2020). Wang et al. (2013) analyzed regional cerebral blood flow-related tissue radioactivity in three-dimensionally reconstructed brains by statistical parametric mapping to explore the neural substrates underlying exercise-based neurorehabilitation. The results of that study highlighted exercise-dependent functional reorganization both in the motor (M1) and limbic circuits (dorsal striatum). In this study, we also found direct evidence of neural plasticity in $\mathrm{M} 1$ cells in PD rats following exercise induction.

Our results confirmed exaggerated beta-range LFP activity in cortical cells of 6-ODHA-lesioned rats, which was effectively eliminated by exercise. Exercise was also able to increase the mean firing rate of PNs; however, the mean firing rates of INs and all cells were not significantly changed. A few factors may have contributed to this result. First, firing rates of PNs and INs vary in different animal models and with different signal acquisition methods (Degenetais et al., 2002; $\mathrm{Xu}$ and Baker, 2018). The majority of previous studies have identified a reduced firing rate in PNs without obvious changes in INs, which may reflect a compensatory change after DA depletion (Pasquereau and Turner, 2011; Shepherd, 2013). Another possibility is that exercise-induced improvements in motor function might modulate the degree of coupling between spikes and LFP oscillations. Multiple studies have shown that spike trains tend to be phase-locked to overactive beta bands in various nuclei and motor cortices (Brazhnik et al., 2012, 2014). In this study, we used multichannel recording technology to simultaneously record spikes and LFPs, which allowed us to analyze the level of synchrony between them. The results showed that the STWA ratio and the percentage of neurons were increased in 6-OHDA-lesioned rats, contributing to movement disorders. Exercise likely induces a critical reduction in the synchronization of spike and beta-range oscillations in M1.

Exercise modalities such as treadmill training, Tai Chi, tango dancing, boxing, and forced cycling incorporating both motor skill learning and aerobic training; they may work synergistically to facilitate neuroplasticity and functional restoration to drive motor and cognitive behavioral improvement in PD. Treadmill can be easily adjusted for exercise elements (include speed and gradient) and its training in subjects with PD has been proved in multiple studies to promote behavioral performances (Da Silva et al., 2018; Dauwan et al., 2021). Forced treadmill and wheel running approaches used to drive movement improvement in patients with PD and finding in animal studies that support the potential mechanism (Ferrazzoli et al., 2018). It should be noted that the dopaminergic depletion of the nigrostriatal system was unable to be reversed by the exercise intervention, as has previously been reported (Sconce et al., 2015; Hood et al., 2016; Shi et al., 2019). This finding might be attributed to the fact that the exercise intervention was started 14 days after the 6-OHDA injection, at which point DA depletion may have been completed. Moreover, the behavioral improvements appeared after 2 or 3 weeks exercise intervention. This timedependence on training might due to the PD rats being in the advanced stage, which affects the cognitive elements and needs more repetitively practice to restoration. Despite the 6-OHDAinduced injury severity, other factors also influence exercisemediated neuroprotection, including the exercise initiated time, the exercise intervention administrated pre or at mild stage of PD may facilitate neuroprotection or an alternative process for behavioral recovery involve neuroplasticity (Jakowec et al., 2016; Ferrazzoli et al., 2018).

Indeed, numerous studies have shown that chronic exercise cannot reverse dopaminergic cell death and that improvements in motor performance are related to other compensatory mechanisms (O'Dell et al., 2007; Churchill et al., 2017; Hou et al., 2017). Moreover, studies with PD animal models have shown that exercise decreases excessive striatal glutamate content, restores glutamatergic receptors, and inhibits glutamatemediated excitotoxicity, thereby contributing to a functional reorganization of the corticostriatal pathway (Van Leeuwen et al., 2010; Toy et al., 2014). Thus, it is likely that the efficacy of the exercise intervention was dependent on plastic changes in the glutamate-mediated corticostriatal pathway, which affected the activity of cortical neurons and led to changes in motor symptoms.

In conclusion, this study provides evidence that a treadmill exercise administration can effectively alleviate motor deficits in PD rats. This exercise-induced behavioral improvement involved the disruption of abnormal neural activity in the M1 region of $\mathrm{PD}$ rats. The synchronization of spike firing and beta oscillations in this model may hold the key to this process, and the functional consequences of this synchrony on exercise-induced neuroplasticity in PD rats need to be verified. 


\section{DATA AVAILABILITY STATEMENT}

The raw data supporting the conclusions of this article will be made available by the authors, without undue reservation.

\section{ETHICS STATEMENT}

The animal study was reviewed and approved by Beijing Normal University Committee for Animal Care.

\section{AUTHOR CONTRIBUTIONS}

$\mathrm{XL}, \mathrm{KS}$, and DQ: conceived and designed the experiments. KS and LH: performed the experiments. KS, LH,

\section{REFERENCES}

Alberts, J. L., Phillips, M., Lowe, M. J., Frankemolle, A., Thota, A., Beall, E. B., et al. (2016). Cortical and motor responses to acute forced exercise in Parkinson's disease. Parkinsonism Relat. Disord. 24, 56-62. doi: 10.1016/j.parkreldis.2016.01.015

Antosik-Wojcinska, A., Swiecicki, L., Dominiak, M., Soltan, E., Bienkowski, P., and Mandat, T. (2017). Impact of STN-DBS on mood, drive, anhedonia, and risk of psychiatric side-effects in the population of PD patients. J. Neurol. Sci. 375, 342-347. doi: 10.1016/j.jns.2017.02.020

Babiloni, C., Del Percio, C., Lizio, R., Noce, G., Lopez, S., Soricelli, A., et al. (2019). Levodopa may affect cortical excitability in Parkinson's disease patients with cognitive deficits as revealed by reduced activity of cortical sources of resting state electroencephalographic rhythms. Neurobiol. Aging 73, 9-20. doi: 10.1016/j.neurobiolaging.2018.08.010

Bonavita, S. (2020). Exercise and Parkinson's disease. Adv. Exp. Med. Biol. 1228, 289-301. doi: 10.1007/978-981-15-1792-1_19

Brazhnik, E., Cruz, A. V., Avila, I., Wahba, M. I., Novikov, N., Ilieva, N. M., et al. (2012). State-dependent spike and local field synchronization between motor cortex and substantia nigra in hemiparkinsonian rats. J. Neurosci. 32, 7869-7880. doi: 10.1523/JNEUROSCI.0943-12.2012

Brazhnik, E., McCoy, A. J., Novikov, N., Hatch, C. E., and Walters, J. R. (2016). Ventral medial thalamic nucleus promotes synchronization of increased high beta oscillatory activity in the basal ganglia-thalamocortical network of the hemiparkinsonian rat. J. Neurosci. 36, 4196-4208. doi: 10.1523/JNEUROSCI.3582-15.2016

Brazhnik, E., Novikov, N., McCoy, A. J., Cruz, A. V., and Walters, J. R. (2014). Functional correlates of exaggerated oscillatory activity in basal ganglia output in hemiparkinsonian rats. Exp. Neurol. 261, 563-577. doi: 10.1016/j.expneurol.2014.07.010

Chen, W., Qiao, D. C., Liu, X. L., and Shi, K. X. (2017). Treadmill exercise improves motor dysfunction and hyperactivity of the corticostriatal glutamatergic pathway in rats with 6-OHDA-induced Parkinson's disease. Neural Plast. 2017:2583910. doi: 10.1155/2017/2583910

Churchill, M. J., Pflibsen, L., Sconce, M. D., Moore, C., Kim, K., and Meshul, C. K. (2017). Exercise in an animal model of Parkinson's disease: motor recovery but not restoration of the nigrostriatal pathway. Neuroscience 359, 224-247. doi: 10.1016/j.neuroscience.2017.07.031

Da Silva, F. C., Iop, R. D. R., de Oliveira, L. C., Boll, A. M., de Alvarenga, J. G. S., Gutierres, P. J., et al. (2018). Effects of physical exercise programs on cognitive function in Parkinson's disease patients: a systematic review of randomized controlled trials of the last 10 years. PLoS ONE 13:e193113. doi: 10.1371/journal.pone.0193113

Dauwan, M., Begemann, M. J. H., Slot, M. I. E., Lee, E. H. M., Scheltens, P., and Sommer, I. E. C. (2021). Physical exercise improves quality of life, depressive symptoms, and cognition across chronic brain disorders: a transdiagnostic systematic review and meta-analysis of randomized controlled trials. J. Neurol. 268, 1222-1246. doi: 10.1007/s00415-019-09493-9 and YP: analyzed the data and wrote the paper. All authors contributed to the article and approved the submitted version.

\section{FUNDING}

This work was supported by the National Natural Science Foundation of China (32000833).

\section{ACKNOWLEDGMENTS}

In addition to the listed authors, assistance was provided by Xiangming Lin, Ru Song, and Gang Zhao. We would like to thank Editage (www.editage.cn) for English language editing.

Degenetais, E., Thierry, A. M., Glowinski, J., and Gioanni, Y. (2002). Electrophysiological properties of pyramidal neurons in the rat prefrontal cortex: an in vivo intracellular recording study. Cereb. Cortex 12, 1-16. doi: $10.1093 /$ cercor/12.1.1

Dejean, C., Gross, C. E., Bioulac, B., and Boraud, T. (2008). Dynamic changes in the cortex-basal ganglia network after dopamine depletion in the rat. $J$. Neurophysiol. 100, 385-396. doi: 10.1152/jn.90466.2008

Denker, M., Roux, S., Linden, H., Diesmann, M., Riehle, A., and Grun, S. (2011). The local field potential reflects surplus spike synchrony. Cereb. Cortex 21, 2681-2695. doi: 10.1093/cercor/bhr040

Duchesne, C., Lungu, O., Nadeau, A., Robillard, M. E., Bore, A., Bobeuf, F. et al. (2015). Enhancing both motor and cognitive functioning in Parkinson's disease: aerobic exercise as a rehabilitative intervention. Brain Cogn. 99, 68-77. doi: 10.1016/j.bandc.2015.07.005

Feng, Y. S., Yang, S. D., Tan, Z. X., Wang, M. M., Xing, Y., Dong, F., et al. (2020). The benefits and mechanisms of exercise training for Parkinson's disease. Life Sci. 245:117345. doi: 10.1016/j.lfs.2020.117345

Ferrazzoli, D., Ortelli, P., Madeo, G., Giladi, N., Petzinger, G. M., and Frazzitta, G. (2018). Basal ganglia and beyond: the interplay between motor and cognitive aspects in Parkinson's disease rehabilitation. Neurosci. Biobehav. Rev. 90 294-308. doi: 10.1016/j.neubiorev.2018.05.007

Fox, S. H., Katzenschlager, R., Lim, S. Y., Barton, B., de Bie, R. M. A., Seppi, K., et al (2018). International Parkinson and Movement Disorder Society evidencebased medicine review: update on treatments for the motor symptoms of Parkinson's disease. Mov. Disord. 33, 1248-1266. doi: 10.1002/mds. 27372

Ginanneschi, F., Messa, L. V., Battisti, C., and Rossi, A. (2021). Changes in corticomotor pathway excitability after exercise training in Parkinson's disease. Neurol. Sci. doi: 10.1007/s10072-020-04960-y. [Epub ahead of print].

Goldberg, J. A., Rokni, U., Boraud, T., Vaadia, E., and Bergman, H. (2004). Spike synchronization in the cortex-basal ganglia networks of parkinsonian primates reflects global dynamics of the local field potentials. J. Neurosci. 24, 6003-6010. doi: 10.1523/JNEUROSCI.4848-03.2004

Hammond, C., Bergman, H., and Brown, P. (2007). Pathological synchronization in Parkinson's disease: networks, models, and treatments. Trends Neurosci. 30, 357-364. doi: 10.1016/j.tins.2007.05.004

Holt, A. B., Kormann, E., Gulberti, A., Potter-Nerger, M., McNamara, C. G., Cagnan, H., et al. (2019). Phase-dependent suppression of beta oscillations in Parkinson's disease patients. J. Neurosci. 39, 1119-1134. doi: 10.1523/JNEUROSCI.1913-18.2018

Hood, R. L., Liguore, W. A., Moore, C., Pflibsen, L., and Meshul, C. K. (2016) Exercise intervention increases spontaneous locomotion but fails to attenuate dopaminergic system loss in a progressive MPTP model in aged mice. Brain Res. 1646, 535-542. doi: 10.1016/j.brainres.2016.06.032

Hou, L. J., Chen, W., Liu, X. L., Qiao, D. C., and Zhou, F. M. (2017). Exerciseinduced neuroprotection of the nigrostriatal dopamine system in Parkinson's disease. Front. Aging Neurosci. 9:35810. doi: 10.3389/fnagi.2017.00358

Huot, P., Johnston, T. H., Koprich, J. B., Fox, S. H., and Brotchie, J. M. (2013). The pharmacology of L-DOPA-induced dyskinesia in 
Parkinson's disease. Pharmacol. Rev. 65, 171-222. doi: 10.1124/pr.111.0 05678

Jakowec, M. W., Wang, Z., Holschneider, D., Beeler, J., and Petzinger, G. M. (2016). Engaging cognitive circuits to promote motor recovery in degenerative disorders. Exercise as a learning modality. J. Hum. Kinet. 52, 35-51. doi: 10.1515/hukin-2015-0192

Jankovic, J. (2008). Parkinson's disease: clinical features and diagnosis. J. Neurol. Neurosurg. Psychiatry 79, 368-376. doi: 10.1136/jnnp.2007.131045

Kalia, L. V., and Lang, A. E. (2015). Parkinson's disease. Lancet 386, 896-912. doi: 10.1016/S0140-6736(14)61393-3

Kuhn, A. A., Kempf, F., Brucke, C., Doyle, L. G., Martinez-Torres, I., Pogosyan, A., et al. (2008). High-frequency stimulation of the subthalamic nucleus suppresses oscillatory beta activity in patients with Parkinson's disease in parallel with improvement in motor performance. J. Neurosci. 28, 6165-6173. doi: 10.1523/JNEUROSCI.0282-08.2008

Lacey, M. G., Gooding-Williams, G., Prokic, E. J., Yamawaki, N., Hall, S. D., Stanford, I. M., et al. (2014). Spike firing and IPSPs in layer V pyramidal neurons during beta oscillations in rat primary motor cortex (M1) in vitro. PLoS ONE 9:e85109. doi: 10.1371/journal.pone.0085109

Leventhal, D. K., Gage, G. J., Schmidt, R., Pettibone, J. R., Case, A. C., and Berke, J. D. (2012). Basal ganglia beta oscillations accompany cue utilization. Neuron 73, 523-536. doi: 10.1016/j.neuron.2011.11.032

Li, Q., Ke, Y., Chan, D. C. W., Qian, Z. M., Yung, K. K. L., Ko, H., et al. (2012). Therapeutic deep brain stimulation in parkinsonian rats directly influences motor cortex. Neuron 76, 1030-1041. doi: 10.1016/j.neuron.2012. 09.032

O’Dell, S. J., Gross, N. B., Fricks, A. N., Casiano, B. D., Nguyen, T. B., and Marshall, J. F. (2007). Running wheel exercise enhances recovery from nigrostriatal dopamine injury without inducing neuroprotection. Neuroscience 144, 1141-1151. doi: 10.1016/j.neuroscience.2006.10.042

Pasquereau, B., and Turner, R. S. (2011). Primary motor cortex of the parkinsonian monkey: differential effects on the spontaneous activity of pyramidal tract-type neurons. Cereb. Cortex 21, 1362-1378. doi: 10.1093/cercor/bhq217

Paxinos, G., and Watson, C. (2007). The Rat Brain in Stereotaxic Coordinates. Amsterdam: Elsevier.

Pedersen, B. K., and Saltin, B. (2015). Exercise as medicine-evidence for prescribing exercise as therapy in 26 different chronic diseases. Scand. J. Med. Sci. Sports 25, 1-72. doi: 10.1111/sms.12581

Pogosyan, A., Gaynor, L. D., Eusebio, A., and Brown, P. (2009). Boosting cortical activity at beta-band frequencies slows movement in humans. Curr. Biol. 19, 1637-1641. doi: 10.1016/j.cub.2009.07.074

Sconce, M. D., Churchill, M. J., Greene, R. E., and Meshul, C. K. (2015). Intervention with exercise restores motor deficits but not nigrostriatal loss in a progressive MPTP mouse model of Parkinson's disease. Neuroscience 299, 156-174. doi: 10.1016/j.neuroscience.2015.04.069

Shepherd, G. M. G. (2013). Corticostriatal connectivity and its role in disease. Nat. Rev. Neurosci. 14, 278-291. doi: 10.1038/nr n3469

Shi, K. X., Liu, X. L., Hou, L. J., Qiao, D. C., and Lin, X. M. (2019). Effects of exercise on mGluR-mediated glutamatergic transmission in the striatum of hemiparkinsonian rats. Neurosci. Lett. 705, 143-150. doi: 10.1016/j.neulet.2019.04.052

Shi, K. X., Liu, X. L., Qiao, D. C., Chen, Y. X., and Zhang, L. T. (2017b). Treadmill exercise modulates abnormal $\beta$ oscillation of M1 in the hemiparkinsonian rat. J. Xian Phys. Educ. Univ. 34, 590-598. doi: 10.16063/j.cnki.issn1001-747x.2017.05.014
Shi, K. X., Liu, X. L., Qiao, D. C., and Hou, L. J. (2017a). Effects of treadmill exercise on spontaneous firing activities of striatal neurons in a rat model of Parkinson's disease. Motor Control 21, 58-71. doi: 10.1123/mc.2015-0065

Stein, E., and Bar-Gad, I. (2013). Beta oscillations in the corticobasal ganglia loop during parkinsonism. Exp. Neurol. 245, 52-59. doi: 10.1016/j.expneurol.2012.07.023

Tajiri, N., Yasuhara, T., Shingo, T., Kondo, A., Yuan, W. J., Kadota, T., et al. (2010). Exercise exerts neuroprotective effects on Parkinson's disease model of rats. Brain Res. 1310, 200-207. doi: 10.1016/j.brainres.2009.10.075

Tinkhauser, G., Torrecillos, F., Duclos, Y., Tan, H., Pogosyan, A., Fischer, P., et al. (2018). Beta burst coupling across the motor circuit in patients with Parkinson's disease. Neurobiol. Dis. 117, 217-225. doi: 10.1016/j.nbd.2018.06.007

Toy, W. A., Petzinger, G. M., Leyshon, B. J., Akopian, G. K., Walsh, J. P., Hoffman, M. V., et al. (2014). Treadmill exercise reverses dendritic spine loss in direct and indirect striatal medium spiny neurons in the 1-methyl-4-phenyl-1,2,3,6tetrahydropyridine (MPTP) mouse model of Parkinson's disease. Neurobiol. Dis. 63, 201-209. doi: 10.1016/j.nbd.2013.11.017

Van Leeuwen, J. E., Petzinger, G. M., Walsh, J. P., Akopian, G. K., Vuckovic, M., and Jakowec, M. W. (2010). Altered AMPA receptor expression with treadmill exercise in the 1-Methyl-4-Phenyl-1,2,3,6-Tetrahydropyridinelesioned mouse model of basal ganglia injury. J. Neurosci. Res. 88, 650-668. doi: 10.1002/jnr.22216

Wang, Z., Guo, Y. M., Myers, K. G., Heintz, R., and Holschneider, D. P. (2015). Recruitment of the prefrontal cortex and cerebellum in Parkinsonian rats following skilled aerobic exercise. Neurobiol. Dis. 77, 71-87. doi: 10.1016/j.nbd.2015.02.020

Wang, Z., Myers, K. G., Guo, Y. M., Ocampo, M. A., Pang, R. D., Jakowec, M. W., et al. (2013). Functional reorganization of motor and limbic circuits after exercise training in a rat model of bilateral parkinsonism. PLOS ONE 8:e80058. doi: 10.1371/journal.pone.0080058

Wiest, C., Tinkhauser, G., Pogosyan, A., Bange, M., Muthuraman, M., Groppa, S., et al. (2020). Local field potential activity dynamics in response to deep brain stimulation of the subthalamic nucleus in Parkinson's disease. Neurobiol. Dis. 143:105019. doi: 10.1016/j.nbd.2020.105019

Wilson, C. J., Higgs, M. H., Simmons, D. V., and Morales, J. C. (2018). Oscillations and spike entrainment. F1000Res. 7:F1000. doi: 10.12688/f1000research.16451.1

$\mathrm{Xu}, \mathrm{W}$., and Baker, S. N. (2018). In vitro characterization of intrinsic properties and local synaptic inputs to pyramidal neurons in macaque primary motor cortex. Eur. J. Neurosci. 48, 2071-2083. doi: 10.1111/ejn.14076

Zhuang, Q. X., Li, G. Y., Li, B., Zhang, C. Z., Zhang, X. Y., Xi, K., et al. (2018). Regularizing firing patterns of rat subthalamic neurons ameliorates parkinsonian motor deficits. J. Clin. Invest. 128, 5413-5427. doi: 10.1172/JCI99986

Conflict of Interest: The authors declare that the research was conducted in the absence of any commercial or financial relationships that could be construed as a potential conflict of interest.

Copyright (c) 2021 Shi, Liu, Hou, Qiao and Peng. This is an open-access article distributed under the terms of the Creative Commons Attribution License (CC BY). The use, distribution or reproduction in other forums is permitted, provided the original author(s) and the copyright owner(s) are credited and that the original publication in this journal is cited, in accordance with accepted academic practice. No use, distribution or reproduction is permitted which does not comply with these terms. 